\title{
Capture flows of funnel-and-gate reactive barriers without gravel packs
}

\author{
H. Klammler ${ }^{1,2,3}$, K. Hatfield ${ }^{1,2}$ \& A. Kacimov ${ }^{4}$ \\ ${ }^{I}$ Department of Civil \& Coastal Engineering, University of Florida, USA \\ ${ }^{2}$ Inter-Disciplinary Program in Hydrologic Science, \\ University of Florida, USA \\ ${ }^{3}$ Department of Environmental Science \& Sustainable Development, \\ Fed. University Bahia, Brazil \\ ${ }^{4}$ Department of Soils, Water \& Agricultural Engineering, \\ Sultan Qaboos University, Oman
}

\begin{abstract}
Permeable reactive barriers (PRBs) are a passive in-situ technology, which is based on the interception and physical, chemical and/or biological remediation of a contaminant plume through installation of reactive material in an aquifer. Previous work of the authors includes analytical approaches in two dimensions (horizontal plane) based on the conformal mapping technique that allows for the determination of the groundwater flow fields and capture zones of PRBs of different types. Solutions assume that the permeability $k_{r}$ of the reactive material itself is high with respect to the surrounding aquifer permeability $k_{a}$ or that highly permeable gravel packs are present to equilibrate the hydraulic heads at the up and down-gradient faces of the reactor. Respective results include a simple relationship $Q(R)$ between capture flow $Q$ and reactor Darcian hydraulic resistance $R$. Based on the same technique, the present work gives an exact solution for funnel-and-gate (FG) and velocity equalization wall (VEW) PRBs without gravel packs for the particular case of $k_{r}=k_{a}$. Furthermore, a numerical finite difference study is performed to show that $Q(R)$ is a good approximation (with errors in the $1 \%$ range of maximum capture flow $Q(0)$ ) for FG and VEW
\end{abstract}


PRBs of arbitrary geometric configurations and arbitrary values of $k_{r} / k_{a}$, even in the absence of highly permeable gravel packs at the reactor entrance and exit faces.

Keywords: PRB, groundwater, aquifer, contaminant plume, passive remediation, conformal mapping, Schwarz-Christoffel.

\section{Introduction}

Subsurface contaminant plumes transported by the (natural or induced) flow of groundwater represent a serious threat to ecosystems and human drinking water supplies. As opposed to hydraulically active pump-and-treat systems for contaminant remediation at the ground surface, [1], permeable reactive barriers (PRBs) are a passive technology, which has been successfully employed for insitu remediation of contaminant plumes through installation of reactive materials in the pathway of a plume, [2]. Actual remediation may hereby be based on physical, chemical and/or biological processes during the residence/travel time of a contaminant particle inside the reactor. Critical parameters in PRB design and operation are (1) the capture flow or width, which determine the portion of groundwater flow and, hence, of the contaminant plume captured for treatment, as well as (2) contaminant residence time inside the reactor, which is directly related to the degree of remediation before initially contaminated groundwater exits the PRB and re-enters the aquifer. A variety of methods exist to optimize these two parameters under different conditions, two of which are represented in fig. 1. In the funnel-and-gate configuration (FG; fig. 1(a); [3]), impermeable funnel arms are deployed laterally extending into the aquifer in order to increase

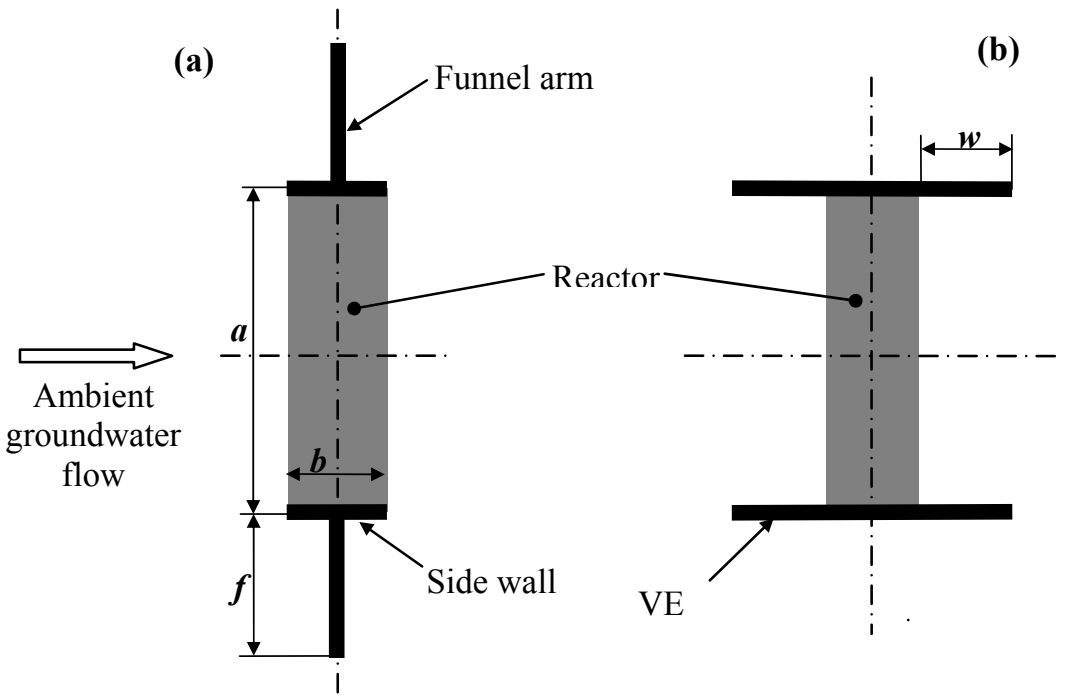

Figure 1: Horizontal cross sections (plan views) of (a) an FG and (b) a VEW PRB. 
capture width for a given reactor. Alternatively, velocity equalization walls (VEWs; fig. 2(b); [4]) may be used to achieve more uniform contaminant residence time distribution across the reactor by avoiding flow singularities (i.e., the blow-up of the magnitude of the hydraulic gradient) near the reactor and, thus, providing largely uniform flow into, across and out of the reactor.

While numerical studies are more abundant in literature, e.g., [3, 4], previous work by the authors, [5-7], investigates different hydraulic aspects of these PRB configurations in a two-dimensional (horizontal) analytical framework by applying the theory of holomorphic functions, in particular, the conformal mapping technique. However, for arbitrary reactor conductivities results are valid only in the presence of highly permeable gravel packs at the up and down-gradient faces of the reactor, which provide for constant hydraulic head distributions throughout the gravel packs and, thus, strictly uniform flow across the reactor. The present work follows this conformal mapping approach and presents an exact analytical solution for the FG and VEW configurations of fig. 1 and the particular case of the reactor hydraulic conductivity $k_{r}[\mathrm{~L} / \mathrm{T}]$ being equal to the surrounding (homogeneous) aquifer conductivity $k_{a}[\mathrm{~L} / \mathrm{T}]$ without, however, assuming the presence of highly permeable gravel packs. Based on this exact solution and results of Klammler and Hatfield [5] for arbitrary $k_{r}$ in the presence of gravel packs, an approximate solution is further presented for the capture flows of FG and VEW PRBs without gravel packs and for arbitrary $k_{r} / k_{a}$. The performance of the approximation is systematically validated against output from a numerical finite difference model (MODFLOW).

\section{Theoretical approach}

\subsection{Exact solution for FG/VEW PRBs without gravel packs and $\boldsymbol{k}_{r}=\boldsymbol{k}_{a}$}

Assuming that vertical flow components in a homogeneous aquifer and PRB reactor may be neglected (i.e., PRB structure stretching over full aquifer thickness) the flow field in the vicinity and inside a FG PRB of $k_{r}=k_{a}$ reduces to two-dimensional flow around the impermeable funnel and side wall structure of fig. 1a. By further assuming that the undisturbed flow field in the aquifer before PRB implementation is uniform with boundaries far away the boundary conditions at the PRB result as illustrated in figs. $2 \mathrm{a}$ and $2 \mathrm{~b}$ for flow in $x$ and $y$ directions (as indicated by the bold arrows), respectively. $q_{x}$ and $q_{y}\left[\mathrm{~L}^{2} / \mathrm{T}\right]$ hereby denote the undisturbed depth integrated ambient groundwater fluxes far from the PRB. Due to symmetry, only the upper half plane is depicted, where thick continuous lines represent impermeable boundaries, thick dotted lines represent constant head boundaries and $z=x+i y[\mathrm{~L}]$ is the complex coordinate system of the physical plane $(i=\sqrt{-1}$ being the imaginary unit). Capital letters denote points (vertices) on the boundary of the flow domain $\left(E_{\infty}\right.$ being the point at infinity), which is symmetric about both the horizontal and vertical axes indicated in fig. 1. Shaded rectangles in figs. 1 and 2 are used to represent the reactor of width $a[\mathrm{~L}]$ and length (in design flow direction) $b[\mathrm{~L}] . f[\mathrm{~L}]$ denotes the length of a funnel arm. Since $k_{r}=k_{a}$ is assumed here, the exact shape of the reactor does not affect the resulting flow field as long as the impermeable funnel 

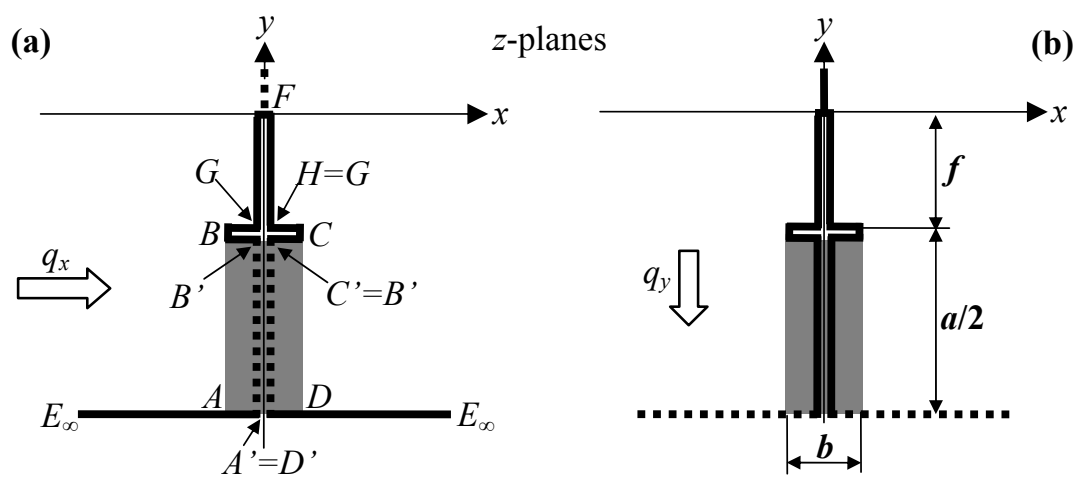

Figure 2: $\quad$ Boundary conditions in the physical $z$-plane for FG PRB with $k_{r}=$ $k_{a}$ and flow in (a) $x$-direction and (b) $y$-direction.

structure is not modified. As a consequence, the VEW configuration of fig. $1 \mathrm{~b}$ does not have to be treated separately and may be regarded as a particular case of the FG configuration with $f=0$, for which flow always remains undisturbed (i.e., uniform) after PRB installation.

By applying the Schwarz-Christoffel function as discussed in Klammler and Hatfield [8] the flow domain above the boundaries of the $z$-plane in figs. 2a and $2 \mathrm{~b}$ may be obtained by conformal mapping of the upper (auxiliary) $\tau_{1}$-half-planes from figs. $3 \mathrm{a}$ and $3 \mathrm{~b}$, respectively, through

$$
Z\left(\tau_{1}\right)=c \int_{0}^{\tau_{1}} \frac{\left(\tau-\tau_{1 B}\right)\left(\tau-\tau_{1 F}\right)\left(\tau-\tau_{1 C}\right)}{\sqrt{\left(\tau-\tau_{1 A \prime}\right)\left(\tau-\tau_{1 B}\right)\left(\tau-\tau_{1 G}\right)\left(\tau-\tau_{1 H}\right)\left(\tau-\tau_{1 C l}\right)\left(\tau-\tau_{\left.1 D^{\prime}\right)}\right.}} d \tau .
$$

$\tau_{l A},[-]$ hereby denotes the coordinate of vertex $A$ ' on the $\eta_{1}$-axis in figs. $3 \mathrm{a}$ and $3 \mathrm{~b}$, for example, and $c[\mathrm{~L}]$ is a positive real scaling constant (no rotation between the $\tau_{l}$ and $z$-planes). Adopting the lower integration limit in eqn. 1 as zero results in $\tau_{I F}=0$ and symmetry further implies $-\tau_{I A},=\tau_{I D},=1 / k,-\tau_{I B},=\tau_{I C},=1,-\tau_{I B}=\tau_{I C}$ and $-\tau_{I G}=\tau_{I H}$ with $c$ accounting for the scaling imposed to achieve $\tau_{I C^{\prime}}=1$. With $k$ [-] being a PRB shape parameter (so-called affix) in the sense of previous work, e.g., [5-7], eqn. 1 may be rewritten as

$$
z\left(\tau_{1}\right)=c \int_{0}^{\tau_{1}} \frac{\left(\tau^{2}-\tau_{1 C}^{2}\right) \tau}{\sqrt{\left(\tau^{2}-\frac{1}{k^{2}}\right)\left(\tau^{2}-1\right)\left(\tau^{2}-\tau_{1 H}^{2}\right)}} d \tau,
$$

which may be reduced to an elliptic integral by substituting $u=\tau^{2}$. The unknown mapping parameters $c, k, \tau_{l C}$ and $\tau_{l H}$ may be determined by constructing a system of four non-linear equations by prescribing the physical coordinates of vertices $A^{\prime}, B^{\prime}, B$ and $G$, for example. However, closed form solutions are generally difficult to obtain (this is known as the Schwarz-Christoffel parameter problem) 
(a) $\tau_{1}$-plane

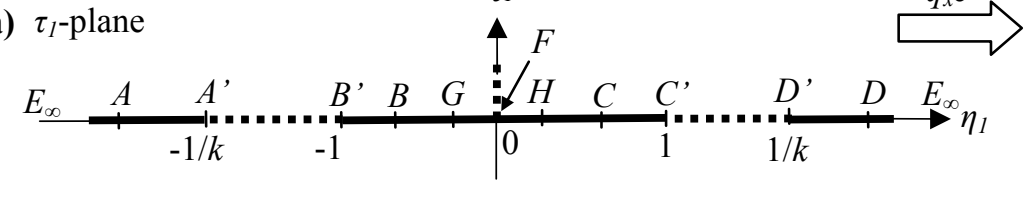

(b) $\tau_{1}$-plane

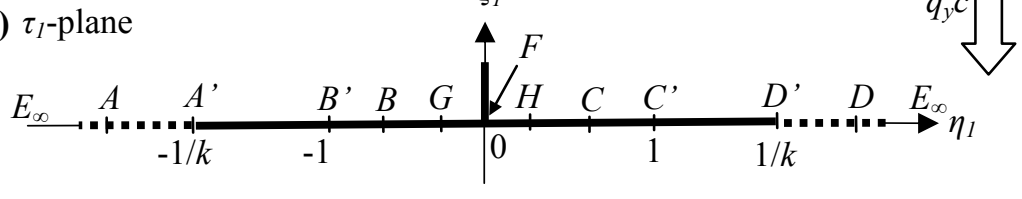

(c) $\tau_{2}$-plane

(d) $\tau_{3}$-plane

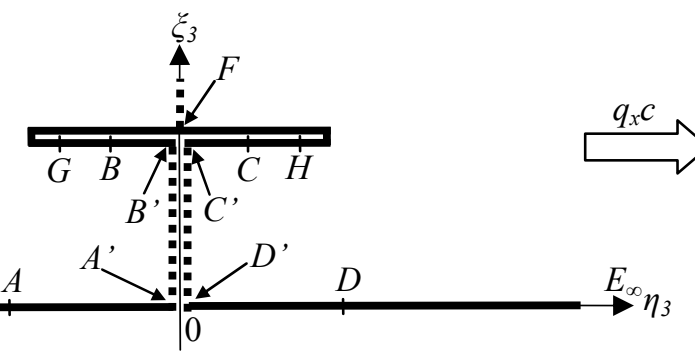

Figure 3: $\quad$ Boundary conditions in the $\tau_{1}, \tau_{2}$ and $\tau_{3}$-planes for flow in $x$ and $y$ directions.

and a Schwarz-Christoffel mapping toolbox [9] for MATLAB is used in the sequel to solve for the mapping parameters and to perform the mapping process (i.e., numerical evaluation and inversion of eqn. 2).

With this, the locations of all vertices in the $\tau_{l}$-plane and the scaling constant $c$ are known. Due to the fact that eqn. 2 stretches the flow domain at infinity by a factor of $c\left(\mathrm{~d} z / \mathrm{d} \tau_{1}=c\right.$ for $\left.\tau_{1} \rightarrow \infty\right)$ and in order to maintain continuity of flow between mapping planes, the magnitudes of the far fields (i.e., ambient fluxes $q_{x}$ and $q_{y}$ ) are adjusted to $c q_{x}$ and $c q_{y}$ in the $\tau_{l}$-plane of figs. $2 \mathrm{a}$ and $2 \mathrm{~b}$, respectively. By applying the mapping function 


$$
\tau_{2}\left(\tau_{1}\right)=\sqrt{\tau_{1}^{2}-\frac{1}{k^{2}}}
$$

and regarding solutions of the square root in the upper $\tau_{2}$-half-plane the flow domain of fig. $3 \mathrm{c}$ is obtained, which contains uniform flow in the $y$-direction. The corresponding complex potential may thus be written as

$$
\Phi_{\mathrm{y}}+i \Psi_{\mathrm{y}}=q_{y} c i \tau_{2}
$$

where $\Phi_{y}$ and $\Psi_{y}\left[\mathrm{~L}^{3} / \mathrm{T}\right]$ are the potential and stream functions for flow in $y$ direction, respectively.

From Klammler and Hatfield [5; their figs. A1a and A1b] it is known that the $\tau_{3}$-plane of fig. $3 \mathrm{~d}$ may be obtained by

$$
\tau_{3}\left(\tau_{1}\right)=\frac{1}{k}\left[E\left(\tau_{1}, k\right)-\frac{E(k)}{K(k)} F\left(\tau_{1}, k\right)\right],
$$

where $K(k)$ and $F\left(\tau_{1}, k\right)$ are the complete and incomplete elliptic integrals of the first kind, respectively, and $E(k)$ and $E\left(\tau_{1}, k\right)$ are the complete and incomplete elliptic integrals of the second kind, respectively. The mapping parameter $k$ is the so-called modulus of the elliptic integrals, [10]. The $\tau_{3}$-plane meets the boundary conditions for uniform flow in $x$-direction and the corresponding complex is

$$
\Phi_{\mathrm{x}}+i \Psi_{\mathrm{x}}=q_{x} c \tau_{3}
$$

The solutions of eqns. 6 and 4 are graphically represented in figs. $4 a$ and $4 b$, respectively, in the form of flow fields for an example PRB of $b / a=0.5$ and $f / a=$ 1. Stream lines of constant $\Psi$ are depicted as continuous, potential lines of constant $\Phi$ as dotted and stream lines delimiting the capture zone are bold. The flow field in fig. $4 \mathrm{c}$ for an arbitrary flow direction $\left(q_{x}=2 q_{y}\right)$ is obtained by superposition (summation) of eqns. 4 and 6 , which is a direct consequence of linearity of the governing Laplace equation for $\Phi$ and $\Psi$. It is seen that the solution not only consists of the flow field in the aquifer, but also the flow field inside the reactor (e.g., $A B C D$ in fig. 2), which may be of interest for computation of contaminant residence times inside the reactor by the method of Klammler et al. [7].

Knowing that an ambient groundwater flow component $q_{y}$ does not drive any flow across the reactor (i.e., across $A^{\prime} B^{\prime}$ in fig. 2), the capture flow $Q\left[\mathrm{~L}^{3} / \mathrm{T}\right]$ is obtained as [5]

$$
Q=\frac{q_{x} c \pi}{k K(k)}
$$

The thick continuous lines in fig. 5 depict the dimensionless capture width $Q /\left(a q_{x}\right)$ based on eqn. 7 as a function of $b / a$ and $f / a$. As intuitively expected, $Q /\left(a q_{x}\right)$ increases as the relative funnel length $f / a$ increases, independent of the reactor size $b / a$. In contrast, independent of fla the dimensionless capture width 

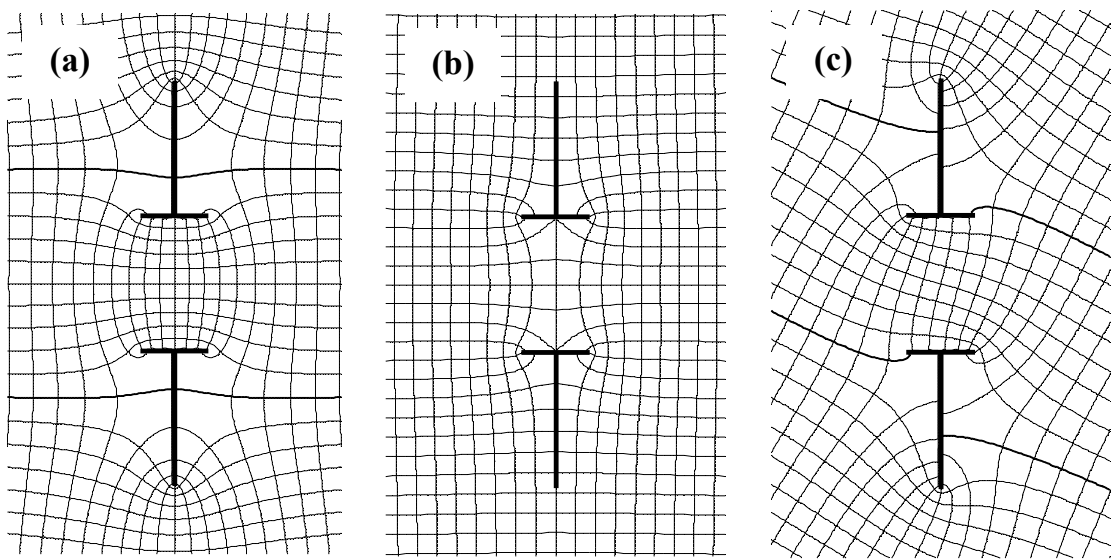

Figure 4: $\quad$ Example of flow nets for flow in (a) $x$-direction, (b) $y$-direction and (c) direction characterized by incident flow of $q_{x}=2 q_{y} . b / a=$ 0.5 and $f / a=1$.

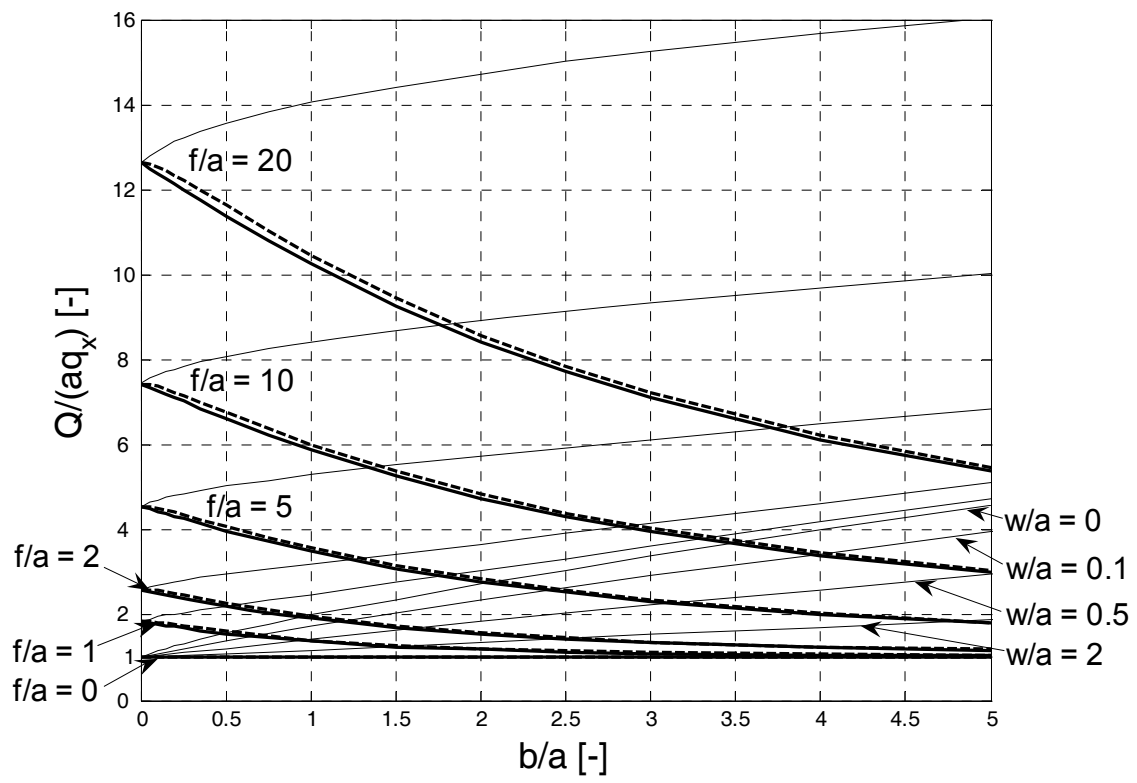

Figure 5: Dimensionless capture width $Q /\left(a q_{x}\right)$ as a function of $b / a$ and $f / a$ for $k_{r}=k_{a}$ and no gravel packs (thick continuous), $k_{r}=k_{a}$ with gravel packs (thick dashed) and $k_{r} \gg k_{a}$ (thin continuous). 
decreases with increasing $b / a$, i.e., as the impermeable side walls become longer. For $f / a<<b / a$ the FG configuration approaches the VEW configuration of fig. $1 \mathrm{~b}$ with $w=0$ (or even $\mathrm{w}>0$ as long as $k_{r}=k_{a}$ ) and $Q /\left(a q_{x}\right)$ approaches unity.

\subsection{Approximate capture flow for FG/VEW PRBs without gravel packs and arbitrary $\boldsymbol{k}_{r} / \boldsymbol{k}_{a}$}

In general, and contrary to the assumption of the previous section, PRB reactors possess hydraulic conductivities different from the aquifer. For example, $k_{r}>k_{a}$ is typically a design goal in order to facilitate plume catchment, i.e., to increase $Q$. On the other hand, $k_{r}<k_{a}$ may be the case if reactive material is installed by direct subsurface injection instead of previous excavation. In addition to the exact solution of the previous section for $k_{r}=k_{a}$, an exact solution is known for $k_{r} \gg k_{a}$, i.e., when the reactor itself is highly permeable. In this case it does not matter whether a gravel pack is actually deployed or not and the solution of Klammler and Hatfield, [5], as illustrated by the thin continuous lines in fig. 5 is directly applicable. Another exact solution may be obtained by the present approach for the absence of gravel packs and $k_{r}<<k_{a}$. While this situation is obviously irrelevant for practice (impermeable reactor) it is noted that the presence or not of gravel packs in this case does not affect the capture flow $Q=$ 0 . Thus, in terms of capture flow, the solution of Klammler and Hatfield, [5], which considers the presence of head equilibrating gravel packs, is exactly valid in the limits of $k_{r} \gg k_{a}$ and $k_{r}<<k_{a}$, independent of the deployment or not of gravel packs.

For the intermediate case of $k_{r}=k_{a}$, fig. 5 compares dimensionless capture flows for no gravel packs (thick continuous lines; previous section) and with gravel packs (thick dashed lines; [5]). Maybe not so unsurprisingly, the agreement is very good with a maximum relative difference of approximately $3 \%$ for $b / a \approx 0.5$ and $f / a \approx 1$. The corresponding flow field for this "worst case" configuration is depicted in fig. $4 \mathrm{a}$, where potential lines confirm a relatively constant head along the reactor-aquifer interfaces (i.e., $A B$ and $C D$ in fig. 2) even without deploying highly permeable gravel packs. Of course, this observation does not hold any longer in the presence of a significant flow component $q_{y}$, which, however, does not affect $Q$. Away from this worst case parameter configuration the influence of gravel packs on $Q$ for $k_{r}=k_{a}$ becomes even smaller and goes to zero for either $b / a, a / b, f / a$ or $a / f$ approaching zero.

Based on the above observations that capture flow $Q$ for FG and VEW PRBs without gravel pack is identical to or well approximated by $Q$ of respective PRB types with gravel packs for the three cases of $k_{r}<<k_{a}, k_{r}=k_{r}$ and $k_{r}>>k_{a}$, we propose that $Q$ in the absence of gravel packs and for arbitrary $k_{r} / k_{a}$ may be approximated by

$$
Q \approx Q_{\max } \frac{K\left(k_{g p}\right)}{K\left(k_{g p}\right)+R K\left(k_{g p}\right)}
$$

as derived by Klammler and Hatfield, [5], for PRBs with gravel packs. $Q_{\max }$ is hereby the maximum capture flow corresponding to $k_{r} \gg k_{a}$ (thin continuous 
lines in fig. 5), $R=b k_{a} /\left(a k_{r}\right)$ [-] is a dimensionless hydraulic resistance of the reactor and $k_{g p}$ [-] is the shape factor for PRBs with gravel packs from Klammler and Hatfield, [5; their fig. 4]. $k_{g p}$ is used as the modulus of the complete elliptic integral or the first kind $K()$ in eqn. 8 with $k_{g p}{ }^{\prime}=\sqrt{1-k_{g p}^{2}}$ [-] being the socalled complementary modulus [10].

\section{Numerical validation and discussion of results}

The validity and accuracy of the approximation postulated in eqn. 8 remains to be evaluated for finite values of $k_{r} / k_{a}$ that are unequal to zero and one. For this purpose, a large (200 × 200 cells) two-dimensional finite difference model (MODFLOW) is used to perform a systematic numerical validation study. 60 model runs without gravel packs are conducted with all possible combinations of the following parameter sets: (1) $k_{r} / k_{a}=\left\{0.1,1,10,100,10^{6}\right\}$, (2) $b / a=\{0.1$, $0.5,2\}$ and (3) $f / a=\{0,1,3\}$ and $w / a=\{0.1\}$. Another 60 model runs are performed for the same PRB geometries (i.e., combinations of $f / a, w / a$ and $b / a$ ), but with gravel packs of conductivity $10^{6} k_{a}$ and with values of $k_{r} / k_{a}$ adjusted such that the thickness of the gravel packs does not affect the reactor hydraulic resistance value $R$. The parameter ranges are chosen to encompass most practical situations as well as the "worst case scenario" for $k_{r}=k_{a}$ from fig. 5. The large value of $k_{r} / k_{a}=10^{6}$ is used to generate a numerical version $Q_{\max , n}$ of the analytical $Q_{\max }$; differences between $Q_{\max , n}$ and $Q_{\max }$ do not exceed 5\% and may be attributed to numerical inaccuracies as well as effects of nearby boundaries required for the numerical method. In order to approximately correct for the latter, numerical capture flows reported in the sequel include a correction factor of $Q_{\max } / Q_{\max , n}$, i.e., an adjustment to honor the known analytical solution for $k_{r}$ $>k_{a}$.

Comparing the numerical output in the presence or not of gravel packs it is observed over the full range of the simulation study that the differences in respective capture flows (counted in the corresponding values of $Q_{\max }$ ) are mostly below $1 \%$ and only exceed $3 \%$ in one case. This is a strong indication that the approximation of eqn. 8 is, in fact, appropriate and well within the error margins of typical field situations. This is further emphasized by fig. 6, which graphically compares analytical and numerical solutions for selected configurations. The continuous and adjacent dashed lines are analogs to respective lines in fig. 5 and represent analytical results from eqns. 7 and 8 , respectively. Numerical output as indicated by the circular (no gravel packs) and star (with gravel packs) markers is in very good agreement, thus further validating eqn. 7 . The remaining dashed lines correspond to $f / a=1$ with $k_{r} / k_{a}=$ 0.1 and 10 (with gravel packs from eqn. 8), which, by comparison with respective numerical output, validate the good performance of eqn. 8 also in the absence of gravel packs down to capture flows as low as approximately 5\% of $Q_{\max }$. Although little relevant for practice, no significant errors may be expected between this and the trivial (but exact) solution for $Q=0$. As a consequence, eqn. 8 may be regarded as exact for the estimation of capture flows in the 


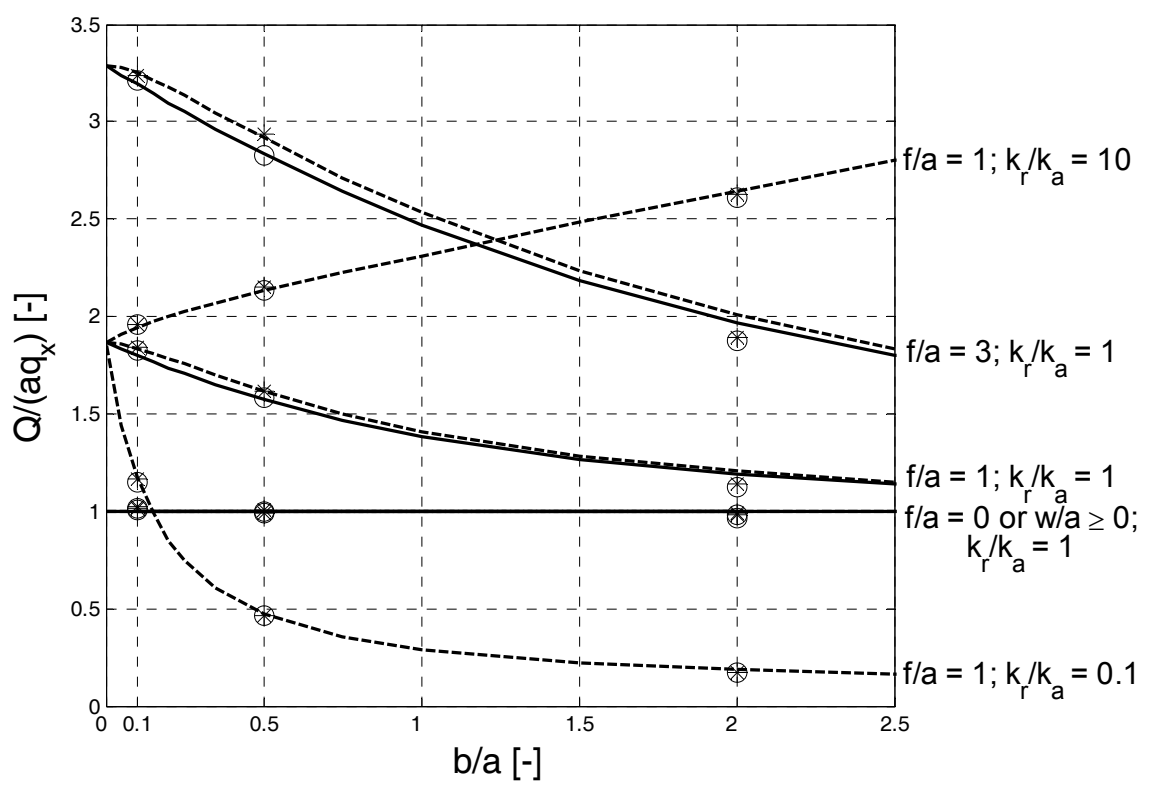

Figure 6: Comparison between analytical (lines) and numerical (markers) results. Continuous lines and circular markers are without gravel packs; dashed lines and star markers are with gravel packs.

presence of highly permeable gravel packs and as a good (errors in the $1 \%$ range of $Q_{\max }$ ) approximation for capture flows of FG and VEW PRBs without gravel packs.

The small difference in capture flows between PRBs with gravel packs and without is a consequence of the fact that the hydraulic head along the reactor faces for flow in $x$-direction is relatively constant even without gravel packs. This is further supported by the fact that differences become even smaller as VEW of increasing length are added, i.e., as the hydraulic head at the reactor faces is even more forced to be constant. The presence of a flow component in $y$ direction does not affect capture flow, which is a pure function of $q_{x}$; capture width, in contrast, only remains unaffected when measured parallel to the $y$ direction. Even though to a quite limited extent, the presence or not of gravel packs does have an influence on capture flows. A closer look at the numerical results (or fig. 5) reveals the rather intuitive fact that gravel packs tend to increase $Q$. However, this is only true for low values of $R$ up to a certain limit and above this limit deployment of gravel packs can actually decrease $Q$. We surmise that this is due to a "potentiating" effect of the low conductivity of gravel packs on the flow regime near the reactor. That is, for low values of $R$ flow generally converges from the aquifer into the reactor and highly permeable gravel packs contribute to increase this flow convergence and, hence, capture flow. In contrast, for high values of $R$ flow divergence, [5], around the reactor may occur and gravel packs contribute to further facilitate this phenomenon, thus, decreasing $Q$ (however, this is not a typical design situation). 


\section{Summary}

Unfortunately, groundwater contamination is currently a wide-spread (global) problem representing a serious threat to ecosystems including ourselves. Once a contaminant source and its down-gradient plume are identified, permeable reactive barriers (PRBs) provide a potential means for partial or full plume remediation with its particular advantages of being passive and in-situ, i.e., treatment occurs without pumping and in the aquifer. This is achieved by installation of a reactive material in the pathway of the contaminant plume, which performs a physical, chemical and / or biological remediation process before releasing the initially contaminated groundwater again into the aquifer. Engineering intervention into the subsurface by constructing a PRB belongs to the class of optimal design problems, with a hydraulic composite assembled as a meta-material (aquifer-wall-gravel pack, reactive material as hydraulic components of similar-dissimilar permeability) placed and structured in a certain manner with respect to the incident groundwater flow. A parameter of fundamental interest in the design and operation of a PRB is its capture flow, i.e., the portion of the ambient groundwater flow captured and treated. Previous analytical approaches by the authors are based on the assumption of the reactive material being much more permeable than the surrounding aquifer or, alternatively, the assumption that highly permeable gravel packs are present at the up and down-gradient faces of the reactor.

In the present work we investigate the influence of gravel packs on capture flows for funnel-and-gate (FG) and velocity equalization wall (VEW) PRBs. An exact analytical solution is presented for the flow field and the capture flow for the particular case of equal aquifer and reactor conductivities and no gravel packs. Graphical inspection of flow fields reveals that the hydraulic heads along the reactor-aquifer interfaces are relatively constant even without gravel packs and that maximum differences in capture flows with respect to the exact solution for gravel packs deployed are not larger than approximately 3\%. Inspired by this, a systematic numerical simulation study considers a wide range of PRB geometries and reactor conductivities to confirm that capture flows of FG and VEW PRBs without gravel packs may accurately be approximated by the respective known solution (eqn. 8) for PRBs with gravel packs from Klammler and Hatfield, [5]. Errors of this approximation are found to be in the $1 \%$ range of the maximum capture flow $Q_{\max }$ for a highly permeable reactor, which is well within the range of uncertainty in field situations.

Evaluation of eqn. 8 is simple and requires determination of $Q_{\max }$ from the thin continuous lines of fig. 5 as a function of the geometric PRB dimensions $b / a$ and $f / a$ (for $\mathrm{FG}$ ) or $w / a$ (for $\mathrm{VEW).} \mathrm{The} \mathrm{shape} \mathrm{parameter} k_{g p}$, also being a function of the geometric PRB dimensions, is directly obtained from Klammler and Hatfield, [5; their " $k$ " in fig. 4]. The values of the complete elliptic integrals of the first kind $K\left(k_{g p}\right)$ and $K\left(k_{g p}^{\prime}\right)$ of modulus $k_{g p}$ and $k_{g p}^{\prime}$, respectively, are easily found from existing look-up tables, e.g., [10], or standard mathematical software. Finally, the dimensionless hydraulic reactor resistance $R$ is a function of the reactor shape and conductivity as defined immediately after eqn. 8 . 


\section{Acknowledgements}

This research was partially funded by the Florida Water Resources Research Center under a grant from the U.S. Department of Interior (06HQGR0079) and NATO CELG grant 983197. The first author gratefully acknowledges support through a fellowship (DCR0001/2009) from the Brazilian National Council for Research and Development (CNPQ) and the Bahia State Research Foundation (FAPESB).

\section{References}

[1] Cunningham, J.A. \& Reinhard, M., Injection-Extraction Treatment Well Pairs: An Alternative to Permeable Reactive Barriers. Ground Water, 40(6), pp. 599-607, 2002.

[2] EPA (Environmental Protection Agency), Capstone Report on the Application, Monitoring, and Performance of Permeable Reactive Barriers for Groundwater Remediation: Volume 1, Performance and Evaluation at Two Sites and Volume 2, Long-Term Monitoring of PRBs: Soil and Groundwater Sampling. EPA/600/R-03/045a and b, 2003.

[3] Starr, R.C. \& Cherry, J.A., In-Situ Remediation of Contaminated Groundwater: The Funnel-and-Gate System. Ground Water, 32(3), pp. 465476, 1994.

[4] Painter, B.D.M., Reactive Barriers: Hydraulic Performance and Design Enhancements. Ground Water, 42(4), pp. 609-617, 2004.

[5] Klammler, H. \& Hatfield, K., Analytical Solutions for the Flow Fields near Funnel-and-Gate Reactive Barriers with Hydraulic Losses. Water Resources Research, 45, W02423, 2009.

[6] Klammler, H., \& Hatfield K., The Problem of Flow-by-Pass at Permeable Reactive Barriers. In: Geo-Environment and Landscape Evolution III, WIT Transactions on the Built Environment, vol. 100, edited by U. Mander, C. A. Brebbia, \& J. F. Martin-Duque, pp. 15-24, 2008.

[7] Klammler, H., Hatfield, K. \& Perminova, I.V., Groundwater and Contaminant Travel Time Distributions near Permeable Reactive Barriers. In: Water Resources Management V. WIT Transactions on Ecology and the Environment, vol. 125, edited by C.A. Brebbia \& V. Popov, pp. 245-256, 2009.

[8] Klammler, H. \& Hatfield, K., Analytical Solutions for Flow Fields near Continuous Wall Reactive Barriers. Journal of Contaminant Hydrology, 98(1-2), pp. 1-14, 2008.

[9] Driscoll, T.A., A MATLAB toolbox for Schwarz-Christoffel Mapping. ACM Transactions on Mathematical Software, 22, pp. 168-186, 1996. (toolbox available under: www.math.udel.edu/ driscoll $/ \mathrm{SC} /$ )

[10] Byrd, P.F., Friedman, M.D., Handbook of Elliptic Integrals for Engineers and Physicists, Springer, Berlin, 1954. 